\title{
INFLUENCE OF POLARIZATION MODE DISPERSION ON THE EFFECT OF CROSS-PHASE MODULATION IN INTENSITY MODULATION-DIRECT DETECTION WDM TRANSMISSION SYSTEM
}

\author{
M. S. ISLAM ${ }^{1 *}$ AND S. P. MAJUMDER ${ }^{2}$ \\ ${ }^{1}$ Institute of Information and Communication Technology \\ ${ }^{2}$ Department of Electrical and Electronic Engineering \\ Bangladesh University of Engineering and Technology, Dhaka-1000, Bangladesh. \\ "E-mail: mdsaifulislam@iict.buet.ac.bd
}

\begin{abstract}
Cross-phase modulation (XPM) changes the state-of-polarization (SOP) of the channels through nonlinear polarization rotation and induces nonlinear time dependent phase shift for polarization components that leads to amplitude modulation of the propagating waves in a wavelength division multiplexing (WDM) system. Due to the presence of birefringence, the angle between the SOP changes randomly and as a result polarization mode dispersion (PMD) causes XPM modulation amplitude fluctuation random in the perturbed channel. In this paper we analytically determine the probability density function of the random angle between the SOP of pump and probe, and evaluate the impact of polarization mode dispersion on XPM in terms of bit error rate, channel spacing etc for a two channel intensity modulation-direct detection WDM system at $10 \mathrm{~Gb} / \mathrm{s}$. It is found that the XPM induced crosstalk is polarization independent for channel spacing greater than $3 \mathrm{~nm}$ or PMD coefficient larger than $2 \mathrm{ps} / \mathrm{km}$. We also investigate the dependence of SOP variance on PMD coefficient and channel spacing.
\end{abstract}

KEYWORDS: Polarization Mode Dispersion, Cross-Phase Modulation, State of Polarization, Birefringence, Bit Error Rate.

\section{INTRODUCTION}

Cross-phase modulation (XPM) is a nonlinear phenomena occurring in optical fibers when two or more optical fields are transmitted through a fiber simultaneously. It has an important impact on the performance of high-speed wavelength division multiplexing (WDM) in optical fiber communication systems [1]. In the last few years, many research works have been carried out on the interaction among fiber nonlinearity, polarization variation and polarization mode dispersion (PMD) [2-8]. Physically, PMD has its origin in the birefringence that is present in any optical fiber. Just like signal distortion due to chromatic dispersion and nonlinearity accumulate along the length of the communication link, so does the polarization and PMD. Polarization fluctuations and PMD become increasingly important as the per-channel data rates have increased and now arguably the most important of the polarization effects. Lin and Agrawal [4], developed a vector theory of XPM in optical fiber and used to investigate the effect of PMD on XPM crosstalk in a WDM system in terms of amplitude of the probe fluctuation induced by a co-propagating pump channel and found that PMD reduces the difference in the average crosstalk level 
between cases of copolarized and orthogonally polarized channels. Zhang et al. [5], experimentally reported that self-phase modulation (SPM) can suppress PMD penalty and XPM-induced polarization scattering can reduce the PMD impairment in single channel and dense WDM transmission system respectively. Here, we focus for simplicity on the pump-probe configuration and neglect pulse broadening induced by group velocity dispersion (GVD), but includes the group velocity mismatch between the pump and probe owing to their different wavelength. We use Jones-matrix formalism for pump- or probe field evolution and Stokes-vector formalism for SOP of pump and probe change on the Poincare sphere.

PMD-induced fluctuation is the relative orientation between the pump and probe SOP which causes the XPM modulation amplitude to be random. In this paper we analytically determined the probability density function (pdf) of the random angle $\theta(z)$ between the pump and probe SOP fluctuation that produce intensity dependent pulse distortion on a propagating signal. Using the pdf we analyzed the impact of PMD on XPM in terms of average bit error rate (BER) for an intensity modulation-direct detection (IM-DD) WDM system at a bit rate of $10 \mathrm{~Gb} / \mathrm{s}$. We also find the variance of the SOP between pump and probe with respect to fiber link and channel spacing. To our knowledge the influence of PMD on XPM in terms of BER is yet to be reported.

\section{SYSTEM MODEL}

The block diagram of a pump-probe configuration WDM system with EDFA in cascade used for theoretical analysis is shown in Fig. 1. The pump and probe are multiplexed by WDM MUX and the composite signal is transmitted through a standard single mode fiber (SMF). To describe the effect of PMD on XPM, we assume that the pump, $\left|A_{1}\right\rangle$ act as channel 1 , which modulates the transmitted $\left(f_{1}\right)$ data from the laser diode at wavelength, $\lambda_{1}$ and the probe, $\left|A_{2}\right\rangle$ act as channel 2, which is a low-power continuous wave (cw) at wavelength, $\lambda_{2}$. The in-line EDFA's are used in cascade to compensate the fiber losses. Finally, the composite signal is demultiplexed at WDM DEMUX and from the modulated carrier; $\lambda_{2}$ original signal $f_{1}$ is recovered through IMDD method.

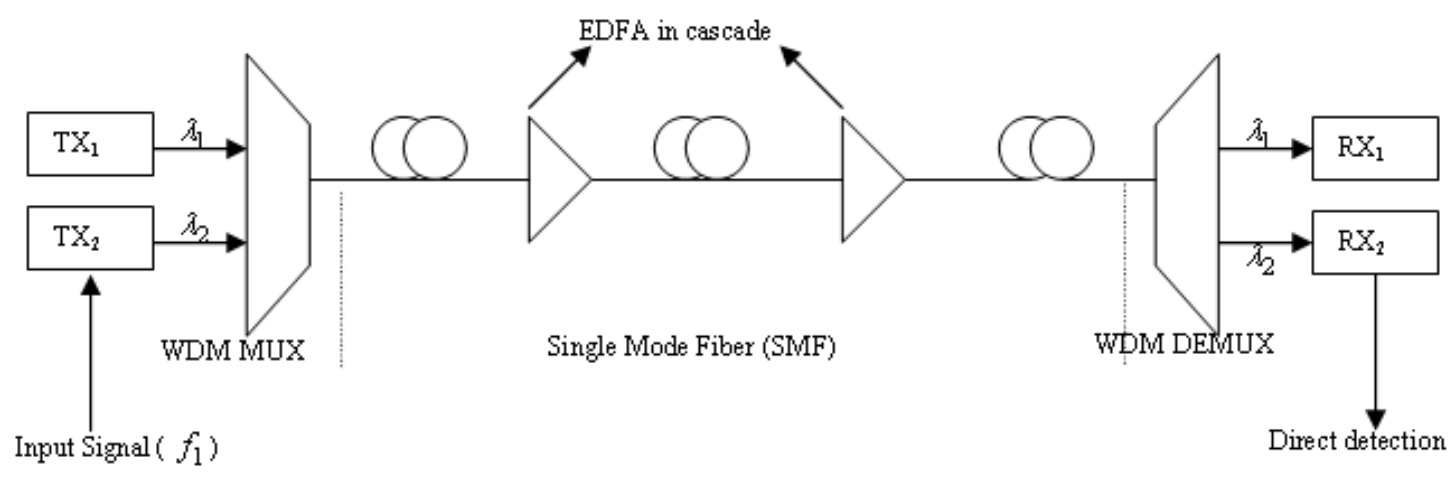

Fig. 1: Block diagram of a 2-channel WDM system with EDFA in cascade. 


\section{THEORETICAL ANALYSIS}

\subsection{Modulation of the Probe Field}

The probe is assumed to be weak enough that the SPM, XPM and intrachannel PMD induced by it can be neglected. Although these effects broadens pulse in each channel, they barely affect the interchannel XPM interaction because the channel spacing typically is much larger than channel bandwidth and the evolution of SOP of the channels is mainly governed by the birefringence. The XPM induces a time dependent phase shift as well as nonlinear polarization rotation on the probe channel that cause the XPM induced modulation amplitude to be random. The cw probe field is modulated by the pump field by the combination of XPM and PMD.

To study the temporal modulation of cw probe field as a consequence of pump field, let us linearize the probe field $\left|A_{2}\right\rangle$ by assuming unperturbed, $\left|A_{20}\right\rangle$ and perturbed, $\left|A_{21}\right\rangle$ probe fields respectively. With these simplifications, we can write the following equations for pump and probe field evolutions as [4],

$$
\begin{aligned}
& \frac{\partial\left|\boldsymbol{A}_{1}\right\rangle}{\partial z}=-\delta \beta_{1} \frac{\partial\left|\boldsymbol{A}_{1}\right\rangle}{\partial \tau}-\frac{i \beta_{21}}{2} \frac{\partial^{2}\left|\boldsymbol{A}_{1}\right\rangle}{\partial \tau^{2}}-\frac{\alpha_{1}}{2}\left|\boldsymbol{A}_{1}\right\rangle-i \varepsilon_{1} P_{0}\left|\boldsymbol{A}_{1}\right\rangle \\
& \frac{\partial\left|\boldsymbol{A}_{20}\right\rangle}{\partial z}=-\frac{\alpha_{2}}{2}\left|\boldsymbol{A}_{20}\right\rangle-\frac{i}{2} \Omega \boldsymbol{b} . \boldsymbol{\sigma}\left|\boldsymbol{A}_{20}\right\rangle \\
& \frac{\partial\left\langle\boldsymbol{A}_{20} \mid \boldsymbol{A}_{21}\right\rangle}{\partial z}=-\frac{i \beta_{22}}{2} \frac{\partial^{2}\left\langle\boldsymbol{A}_{20} \mid \boldsymbol{A}_{21}\right\rangle}{\partial \tau^{2}}-\alpha_{2}\left\langle\boldsymbol{A}_{20} \mid \boldsymbol{A}_{21}\right\rangle+\frac{i \varepsilon_{2}}{2} P_{0} S_{0}[3+\cos \theta(z)]
\end{aligned}
$$

where, $P_{0}=\left\langle\boldsymbol{A}_{1} \mid \boldsymbol{A}_{1}\right\rangle$ and $\boldsymbol{S}_{0}=\left\langle\boldsymbol{A}_{20} \mid \boldsymbol{A}_{20}\right\rangle$ are the pump and unperturbed probe power; $\hat{P}=\left\langle\boldsymbol{A}_{1}|\boldsymbol{\sigma}| \boldsymbol{A}_{1}\right\rangle / P_{0}$ and $\hat{\mathrm{S}}=\left\langle\boldsymbol{A}_{20}|\boldsymbol{\sigma}| \boldsymbol{A}_{20}\right\rangle / \boldsymbol{S}_{0}$ are the unit vector representing the SOP of the pump and unperturbed probe on the Poincare sphere; $\Omega=\left(\omega_{2}-\omega_{1}\right)$ is the channel spacing, $\varepsilon_{1}=8 / 9 \gamma_{1}$ and $\varepsilon_{2}=8 / 9 \gamma_{2}$ are the effective nonlinear parameters, $\theta(z)=\hat{P} . \hat{S}(z)$ is the random angle between the pump and probe SOP, $\delta \beta_{1}=\left(\beta_{11}-\beta_{12}\right)$ is the group velocity mismatch between the two channels and $\tau=\left(t-\beta_{12} z\right)$ is the reduced temporal variable.

From (1) we see that the pump polarization $\hat{P}$ remain fixed in the rotating frame. However, PMD changes the relative orientation between the pump and probe stokes vectors at a rate dictated by the magnitude of channel spacing and relative birefringence $(\Omega \boldsymbol{b})$. The total optical power of the probe field is given by,

$$
\left\langle A_{2} \mid A_{2}\right\rangle \approx\left\langle A_{20} \mid A_{20}\right\rangle+\left[\left\langle A_{20} \mid A_{21}\right\rangle+\text { c.c. }\right] \equiv S_{0}+\delta S_{0}
$$

where $\delta \boldsymbol{S}_{\boldsymbol{0}}$ is the modulation amplitude, which is a measure of the XPM induced crosstalk. PMD randomly changes the angle between $\hat{\mathrm{P}}$ and $\hat{\mathrm{S}}(\mathrm{z})$ along the fiber and thus probe field modulation amplitude becomes a random quantity. Solving equation (3) 
analytically and introducing the normalized modulation amplitude as, $\tilde{\delta}_{X}(\omega)=\delta \widetilde{S}_{0}(L, \omega) / S_{0}(L)$; we obtain,

$$
\tilde{\delta}_{X}(\omega)=-\int_{0}^{L} \varepsilon_{2}(z) \tilde{\boldsymbol{P}}_{0}(z, \omega)[3+\cos \theta(z)] \sin \left[\frac{1}{2} \int_{z}^{L} \omega^{2} \beta_{22}\left(z_{1}\right) d z_{1}\right] d z
$$

where a tilde denotes the Fourier transform and $\widetilde{\boldsymbol{P}}_{\mathbf{0}}(z, \omega)$ is the Fourier spectrum of the pump power at a distance $z$ inside the fiber and $\beta_{22}$ is the GVD coefficient of the pump.

\subsection{Pdf of $\theta(z)$, SOP and Crosstalk Variance}

Since the pump polarization $\hat{P}$ remains constant and the random variation of $\hat{S}(z)$ (unit vector of SOP of the probe) will be responsible for the randomness of $\theta(z)$. Thus to find the pdf of the angle $\theta(z)$ between pump and probe, we assume that it is driven by the white noise process and can be written as,

$$
\begin{array}{ll} 
& \frac{d \theta}{d z}=\hat{S}(z) \\
\text { where, } \quad & \langle\hat{S}(z)\rangle=0 \\
& \left\langle\hat{S}\left(z_{1}\right) \hat{S}\left(z_{2}\right)\right\rangle=\eta^{2} \delta\left(z_{2}-z_{1}\right)
\end{array}
$$

where $\eta=1 / L_{d}=D_{p}^{2} \Omega^{2} / 3 ; \Omega=\omega_{2}-\omega_{1}$ is the channel spacing and $D_{p}$ is the PMD parameter. This model captures all the essentials of the more realistic case in which both the angle $\theta(z)$ and birefringence vary randomly. From equation (8) a diffusion equation for the probability distribution of $\theta(z)$ or simply $\boldsymbol{\Theta}$ can be obtained [9],

$$
\frac{\partial p}{\partial z}=\frac{1}{2} \eta^{2} \frac{\partial^{2} p}{\partial \theta^{2}}
$$

where $p(\theta, z)$ is $2 \pi$ periodic in $\theta$ and $p(0, z)=\delta\left(\theta-\theta_{0}\right) . \theta_{0}$ is the value of $\theta$ at $z=0, \theta_{0}$ is the relative angle between the pump and probe SOPs at the input end of the fiber. By solving the equation (9) we get the pdf $\theta(z)$ and expressed as,

$$
p(\theta, z)=\frac{1}{2 \pi}+\frac{1}{\pi} \sum_{m=1}^{\infty} \exp \left(-\frac{1}{2} \eta^{2} m^{2} z\right) \cos m\left(\theta-\theta_{0}\right)
$$

The SOP of the pump and probe depends on the power and bit pattern of the pump. The SOP fluctuates with time because of its doubly in nature. In the absence of residual birefringence, randomness comes only from the bit pattern. The SOP of pump and probe variance can be obtained by taking the inverse Fourier transform of equation (8) along the fiber link length $L$, 


$$
\begin{aligned}
\sigma_{\text {sop }}^{2} & =\int_{0}^{L} d z_{1} \int_{0}^{L} d z_{2} F^{-1}\left\{\left\langle\hat{S}\left(z_{1}\right) \hat{S}\left(z_{2}\right)\right\rangle\right\} \\
& =\frac{1}{4 \pi^{2}} \int_{-\infty}^{\infty} d \omega_{1} \int_{-\infty}^{\infty} d \omega_{2}\left[\int_{0}^{L} d z_{1} \int_{0}^{L} d z_{2} \eta^{2} \delta\left(z_{1}-z_{2}\right)\right]
\end{aligned}
$$

To find the $\widetilde{\boldsymbol{P}}_{\mathbf{0}}(z, \omega)$, we assume that the effects of dispersion and nonlinearities do not significantly change the pulse shape of the pump channel along the fiber and analytically solving(1), we then obtain,

$$
\widetilde{\boldsymbol{P}}_{0}(z, \omega)=\tilde{\boldsymbol{P}}_{0}(0, \omega) \exp \left\{\int_{0}^{z}\left[-\alpha_{1}\left(z_{1}\right)+i \omega \delta \beta_{1}\left(z_{1}\right)\right] d z_{1}\right\}
$$

Substituting this expression in (5), we obtain the following analytic result for the XPMinduced crosstalk,

$$
\tilde{\delta}_{X}(\omega)=\tilde{\boldsymbol{P}}_{0}(0, \omega) \int_{0}^{L}[3+\cos \theta(z)] F(z) d z
$$

where the function $F(z)$ takes into account loss and dispersion variation along the fiber link and is given by,

$$
F(z)=-\varepsilon_{2}(z) \exp \left\{\int_{0}^{z}\left[-\alpha_{1}\left(z_{1}\right)+i \omega \delta \beta_{1}\left(z_{1}\right)\right] d z_{1}\right\} \sin \left[\frac{1}{2} \int_{z}^{L} \omega^{2} \beta_{22}\left(z_{1}\right) d z_{1}\right] d z
$$

The crosstalk level changes with time depending on the bit pattern in the pump channel. Thus, $\delta_{X}(\tau)$ fluctuates with time and the crosstalk variance, also called XPM-induced interference can given by,

$$
\begin{aligned}
\sigma_{m}^{2} & \equiv \frac{1}{T} \int_{-\frac{T}{2}}^{\frac{T}{2}}\left[\delta_{X}(\tau)\right]^{2} d \tau \\
& =\frac{1}{4 \pi^{2}} \int_{-\infty}^{\infty} d \omega_{1} \int_{-\infty}^{\infty} d \omega_{2} \tilde{\delta}_{X}\left(\omega_{1}\right) \tilde{\delta}_{X}^{*}\left(\omega_{2}\right) \operatorname{sinc}\left[\frac{\left(\omega_{1}-\omega_{2}\right) T}{2}\right]
\end{aligned}
$$

where, $T$ is the time interval of measurement. Usually the measurement time $T$ is small compared with the fluctuation time of PMD and birefringence fluctuations remain frozen during measurement.

\subsection{Bit Error Rate (BER) Expression}

The output noise contributed by the photodetector quantum shot noise, the receiver thermal noise, the interferometric noise due to input intensity fluctuation. The different spectral densities of the noises are: 


$$
\begin{aligned}
& S_{p d}(f)=2 R_{d} P_{s}=2 e\langle i\rangle B W \\
& S_{t h}(f)=i_{t h}^{2}=\frac{4 K T B W}{R_{L}} \\
& S_{p d i}(f)=0.5 R_{d}^{2} P_{s}^{2}\left[S_{x}(f)-\bar{x}^{2} \delta(f)\right]
\end{aligned}
$$

where $S_{x}(f)$ and $\bar{x}$ represent the PSD and the mean value of $x(t)$ respectively and $\delta(f)$ is a delta function in frequency. Denoting the total noise power spectral density by,

$$
S_{n}(f)=S_{p d}(f)+S_{t h}(f)+S_{p d i}(f)
$$

The total noise power (noise variance) at the receiver output can be obtained as,

$$
\sigma_{n}^{2}=\int_{-\infty}^{\infty} S_{n}(f)\left|H_{R}(f)\right|^{2} d f
$$

where, $H_{R}(f)$ is the transfer function of a Gaussian low-pass filter in the receiver of 3-dB bandwidth equal to $0.75 \mathrm{~B}$. The signal to crosstalk noise ratio (SCNR) can be written as,

$$
S C N R=\frac{\left(R_{d} S_{0}\right)^{2}}{\sigma_{m}^{2}+\sigma_{n}^{2}}
$$

where $\sigma_{m}^{2}$ crosstalk variance due to PMD, $R_{d}$ is the responsivity and $S_{0}$ is the probe power and $I_{s}=R_{d} S_{0}$. Thus,

$$
\operatorname{SCNR}\left(\text { for angle } \theta_{0}\right)=\frac{I_{s}{ }^{2}}{\sigma_{m}^{2}\left(\theta_{0}\right)+\sigma_{n}^{2}}
$$

For a given value of $\theta_{0}$, the conditional BER can be expressed as

$$
\operatorname{BER}\left(\theta_{0}\right)=0.5 \operatorname{erfc}\left(\frac{\sqrt{\operatorname{SCNR}\left(\text { for angle } \theta_{0}\right)}}{\sqrt{2}}\right)
$$

Because of environmental changes (i.e., temperature, pressure, vibration, stress, twisting etc.), PMD fluctuates randomly. Generally, the impact of PMD fluctuations as well as the variation of angle, $\theta(z)$ between the pump and probe SOP typically occurs on a time scale of milliseconds. Thus the average BER is given by,

$$
B E R=\int_{-\pi}^{\pi} B E R\left(\theta_{0}\right) p(\theta, z) d \theta
$$




\section{RESULTS AND DISCUSSION}

Following the analytical approach, we investigate the influence of PMD on XPM of the pump-probe configuration of IM-DD WDM system at a bit rate of $10 \mathrm{~Gb} / \mathrm{s}$. The pdf of the random polarization angle between pump and probe is evaluated at various length of single mode fiber and shown in Fig. 2. From the figure, it is found that for particular channel spacing the pdf is a delta function at $z=0$ and becomes flat as the link length increases. It is observed that for any lightwave system of fiber length much larger than the diffusion length ( $h_{E}=280 \mathrm{~m}-1.5 \mathrm{~km}$ ), pdf become Gaussian like distribution.

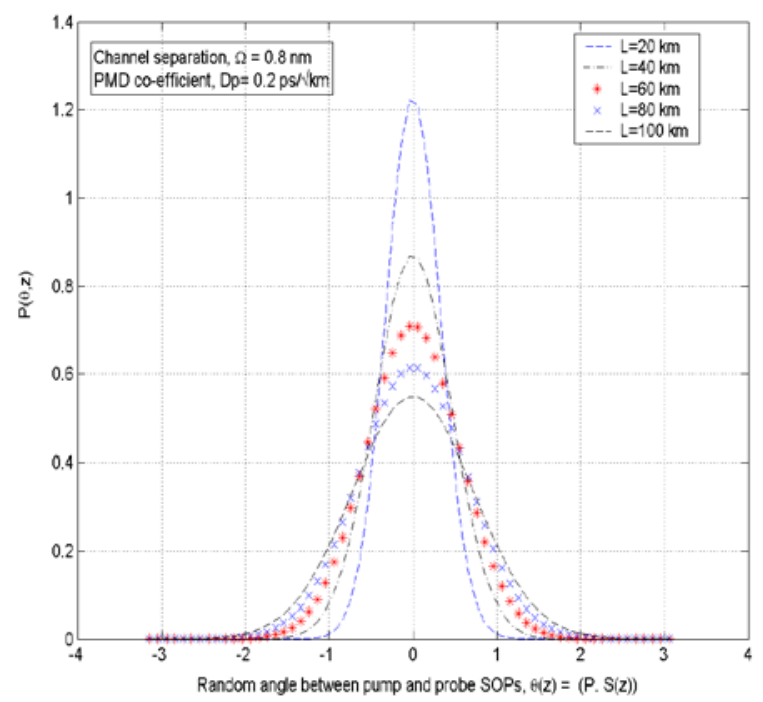

Fig. 2: Plots of pdf of $\theta(z)$ at different length of fiber link.

Figure 3 shows the variance of pump and probe polarization SOP versus fiber link length for different PMD coefficient. It is noticed that as the PMD coefficient increases, the variance of SOP also increases linearly. Thus the fibers which have higher values of PMD (usually older fiber) will subject to larger amount of amplitude modulation fluctuation.

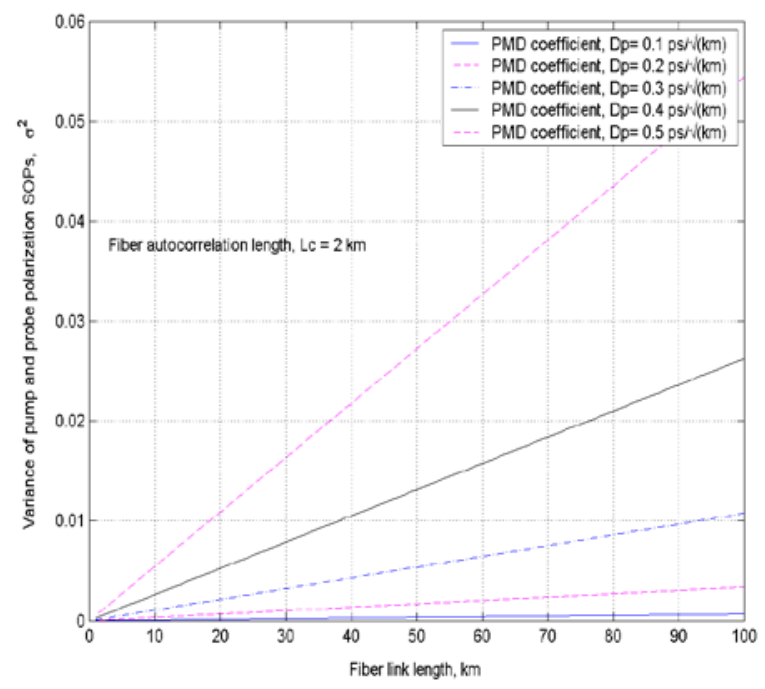

Fig. 3: Plots of variance of SOP against fiber link length for different PMD parameters. 
The average BER versus pump power is plotted in Fig. 4. It is observed that interaction of PMD and XPM increases as the channel spacing decreases for a specific pump power and system suffers a large amount of BER. The significant dependence of XPM effect on BER as a function of channel spacing comes from the fact that the PMD diffusion length is inversely proportional to the square of the channel spacing. However, at increased pump power the BER increases and when pump power is about $16 \mathrm{dBm}$ the BER curves makes a floor at $10^{-1}$ irrespective of channel spacing.

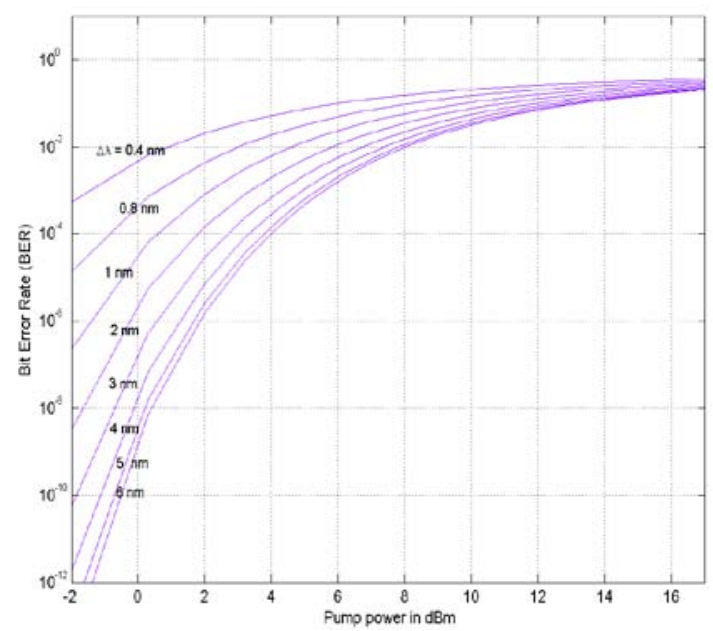

Fig. 4: Plots of average BER against pump power for different channel spacing.

The plots of average BER versus probe power is shown in Fig.5. It is seen that at high pump and probe power the BER performance of the fiber link deteriorates sharply. This happens due to the strong interaction of PMD and XPM on each other channel. For example, when the pump power is $-15 \mathrm{dBm}$, we can achieve a BER of $10^{-9}$ for a $6.25 \mathrm{dBm}$ probe power. Keeping the probe power same, it is found that at $25 \mathrm{dBm}$ pump power the BER drops to a value of $10^{-1}$ only. This happens due to the higher modulation amplitude fluctuation as a result of high pump power.

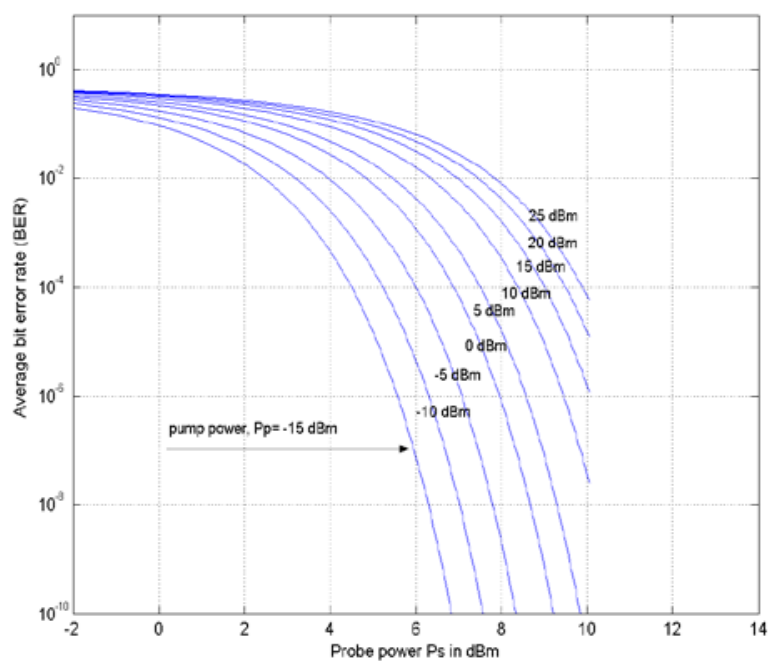

Fig. 5: Plots of average BER vs probe power for different pump power. 
Figure 6 depicts the variance of SOP as a function of channel spacing for different length. For smaller channel spacing and short length of fiber link the variance is very negligible. As the link length increases and so does the channel spacing, the variances increases exponentially for larger values of channel spacing.

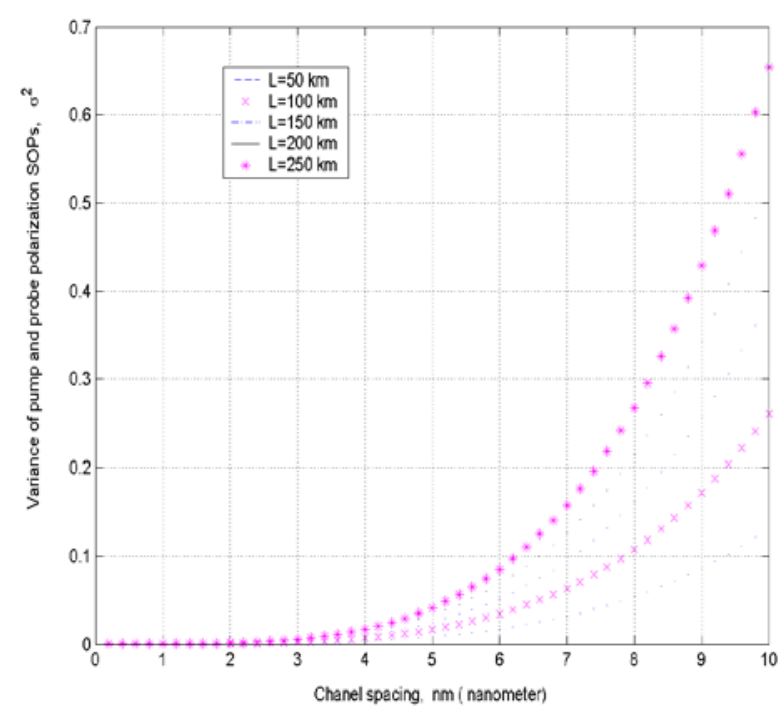

Fig. 6: Plots of variance of SOP against channel spacing ( $\Delta \lambda$ ) for different link length.

\section{CONCLUSION}

An analytical approach is used to investigate the effect of PMD and XPM interaction in a two channel pump-probe configuration WDM system using SMF. We determine the probability distribution function of the random SOP angle between the pump and probe in an IM-DD WDM system, which causes the XPM induced modulation amplitude to be random. The strength of the XPM effect is strongly influenced by the evolution of lightpolarization of the WDM carriers. Our results show that the XPM induced crosstalk becomes polarization independent when channel spacing is large (i.e., $\Delta \lambda>3 \mathrm{~nm}$ ) or when the fiber has a relatively large value of PMD coefficient (i.e., $D_{p}>2 \mathrm{ps} / \sqrt{\mathrm{km}}$ ) . Thus at relatively high pump the combination of nonlinearity and PMD can limit the performance of a dense WDM fiber link significantly.

\section{ACKNOWLEDGEMENT}

This research work has been carried out as a part of Ph.D Thesis work in the Department of Electrical and Electrical Engineering (EEE) of Bangladesh University of Engineering and Technology (BUET), Dhaka, Bangladesh. 


\section{REFERENCES}

[1] P. I. Killey, H. J. Thiele, V. Mikhailove and P. Bayvel, "Prediction of transmission penalties due to cross-phase modulation in WDM systems using simplified technique', IEEE Photon. Technol. Lett., Vol.12, no.7, 804 - 806, 2007.

[2] N. Hanik "Influence of polarization mode dispersion on the effect of cross-talk modulation in optical WDM transmission”, Proc. of the 6th International Conference on Transparent Optical Networks (ICTON’04), Vol.1, 90-194, 2004.

[3] A. Bononi, A. Vanocci, A. Orlandini, E. Corbel, S. Lanne and S. Bigo, "Degree of polarization degradation due to cross-phase modulation and its impact on polarization mode dispersion”, J. Lightwave Technol., Vol. 21, no.9, 1903-1913, 2003.

[4] Q. Lin and G. P. Agrawal, "Effects of polarization mode dispersion on cross-phase modulation in dispersion managed wavelength-division-multiplexed systems”, J. Lightwave Technol., Vol. 22, no. 4, 977-987, 2004.

[5] G. Zhang, J. T. Stango, X. Zhang and C. Xie, "Impact of fiber nonlinearity on PMD penalty in DWDM transmission systems,” IEEE Photon. Technol. Lett., Vol.17, no. 2, 501-503, 2005.

[6] C. R. Menyuk and B. S. Mark, "Interaction of polarization mode dispersion and nonlinearity in optical fiber transmission systems", J. Lightwave Technol., Vol. 24, no.7, 2806-2826, 2006.

[7] R. Khosravani, Y. Xie, L. -S. Yan, A. E. Willner and C. R. Menyuk, "Bit pattern dependent polarization rotation in the first-order PMD-compensated WDM systems", Optics Communications, 191-196, 2005.

[8] L. Moller, Y. Su, G. Raybon, S. Chandrasekar and L. L. Buhl, "Penalty interference of nonlinear intra-channel effect and PMD in ultra-high speed TDM systems", Electron Lett., Vol.38, no.6, 281-283, 2002.

[9] P. K. A Wai and C. R. Menyuk, "Polarization mode dispersion, decorrelation and diffusion in optical fibers with randomly varying birefringence," J. Lightwave Technol., vol.14, no.2, 148-157, 1996. 